\title{
Diagnóstico e tratamento de glomerulonefrite pós-infecciosa - revisão narrativa
}

\author{
Diagnosis and treatment of post-infectious glomerulonephritis - narrative review \\ Diagnóstico y tratamiento de la glomerulonefritis posinfecciosa - revisión narrativa \\ Jorge Luiz dos Santos Pereira ${ }^{1 *}$, Rodolfo Leone de Andrade ${ }^{1}$, Carolina Tofolo¹.
}

\begin{abstract}
RESUMO
Objetivo: Revisar a literatura atualizando os avanços obtidos no diagnóstico e tratamento de glomerulonefrite pós estreptocócica (GNPE). Revisão bibliográfica: A GNPE trata-se de uma sequela tardia, não superativa, de infecção estreptococcia e geralmente envolve manifestações clínicas bastante variáveis, desde formas leves ou assintomáticas até casos sintomáticos mais graves. Pode apresentar incidência endêmica ou epidêmica, sendo rara em menores de 2 anos e mais frequente em crianças com 7 a 12 anos de idade. Cada caso precisa ser analisado de forma específica em termos de tratamento, considerando vários fatores: quadro clínico apresentado, idade, presença ou não de complicações e até mesmo as condições socioculturais e econômicas do paciente. Pesquisas sobre biomarcadores para identificação de pacientes e de vacinas têm sido propostas e conduzidas, porém ainda não estão disponibilizados para atender especialmente a indivíduos que vivem em áreas endêmicas. Considerações finais: Os avanços obtidos nos últimos anos em termos de diagnóstico diferencial e tratamento da GNPE podem auxiliar os profissionais de saúde e as autoridades a vencer este desafio e na adoção de políticas públicas que atuem mais na prevenção das doenças precursoras, tais como faringite e infecções de pele.
\end{abstract}

Palavras-chave: Glomerulonefrite, Glomerulonefrite pós-estreptocócica, Doença renal.

\begin{abstract}
Objective: To review the literature updating the advances obtained in the diagnosis and treatment of poststreptococcal glomerulonephritis (GNPE). Literature review: GNPE is a late, non-overactive sequel of streptococcy and usually involves quite variable clinical manifestations, from mild or asymptomatic forms to more severe symptomatic cases. It can present endemic or epidemic incidence, being rare in children under 2 years old and more frequent in children aged 7 to 12 years old. Each case needs to be analyzed specifically in terms of treatment, considering several factors: clinical presentation, age, presence or absence of complications and even the socio-cultural and economic conditions of the patient. Research on biomarkers for the identification of patients and vaccines has been proposed and conducted, but they are not yet available to serve especially individuals living in endemic areas. Final considerations: The advances obtained in recent years in terms of differential diagnosis and treatment of GNPE can help health professionals and authorities to overcome this challenge and in the adoption of public policies that act more in the prevention of precursor diseases, such as pharyngitis and skin infections.
\end{abstract}

Keywords: Glomerulonephritis, Post-streptococcal glomerulonephritis, Kidney disease.

\section{RESUMEN}

Objetivo: Revisar la literatura actualizando los avances obtenidos en el diagnóstico y tratamiento de la glomerulonefritis post-estreptocócica (GNPE). Revisión bibliográfica: GNPE es una secuela tardía, no hiperactiva, de estreptococos y generalmente involucra manifestaciones clínicas bastante variables, desde formas leves o asintomáticas hasta casos sintomáticos más severos. Puede presentar incidencia endémica o epidémica, siendo rara en niños menores de 2 años y más frecuente en niños de 7 a 12 años. Cada caso debe analizarse de manera específica en términos de tratamiento, considerando varios factores: presentación clínica, edad, presencia o ausencia de complicaciones e incluso las condiciones socioculturales y económicas del paciente. Se han propuesto y realizado investigaciones sobre biomarcadores para la identificación de

1 Universidade de Franca (UNIFRAN), Franca - SP. *E-mail: rodolfolda16@gmail.com 
pacientes y vacunas, pero aún no están disponibles para atender especialmente a las personas que viven en áreas endémicas. Consideraciones finales: Los avances obtenidos en los últimos años en términos de diagnóstico diferencial y tratamiento de GNPE pueden ayudar Los profesionales de la salud y las autoridades para superar este desafío y en la adopción de políticas públicas que actúen más en la prevención de enfermedades precursoras, como la faringitis y las infecciones de la piel.

Palabras clave: Glomerulonefritis, Glomerulonefritis post-estreptocócica, Enfermedad renal.

\section{INTRODUÇÃO}

A glomerulonefrite pós-estreptocócica trata-se de uma sequela tardia, não supurativa, de infecção estreptococcia prévia em vias aéreas superiores (faringo amigdalite) ou infecções de pele (piodermite) causadas por cepas nefritogênicas de estreptococo beta hemolítico do grupo A de Lancefield e outros agentes infecciosos. Com os avanços tecnológicos e estudo de diversas etiologias de glomerulonefrite na literatura, é destaque ressaltar que a grande maioria dos tipos é imunomediada tendo um componente autoimune, em que é associada a influência do ambiente onde o indivíduo vive a fatores de risco genético, cursando com lesões diferenciadas e acometimento glomerular, muitas vezes bilateral dos rins (COUSER WG, 2016; RAMANATHAN G, et al., 2017).

A presente revisão de literatura apresenta um enfoque específico e atualizado da glomerulonefrite pósestreptocócica (GNPE), por ser um dos tipos de glomerulopatias mais comumente encontrado no cenário médico, além de ser considerada uma das patologias renais com os relatos mais antigos. Geralmente evolui com manifestações clínicas bastante variáveis, desde formas leves ou assintomáticas até casos sintomáticos mais graves. Pode apresentar incidência endêmica ou epidêmica, sendo rara em menores de 2 anos e mais frequente em crianças com 7 a 12 anos de idade, com destaque pelo predomínio no sexo masculino. O quadro clínico típico é marcado por alterações na função renal, o que pode ser caracterizado pela chamada síndrome nefrítica que se define pelos achados de proteinúria, edema, causados pela inflamação glomerular generalizada e bilateral. Entretanto, a doença também pode evoluir para quadros clínicos de maior gravidade e se apresentar com complicações a serem consideradas: hipervolemia com edema agudo pulmonar, crise hipertensiva com encefalopatia e convulsões, e insuficiência renal aguda (IRA), podendo levar a necessidade de diálise de urgência (HAHN RG e FORMAN TA, 2005; RIBEIRO NETO JPM e PONTUAL MP, 2014; SOARES PA, et al., 2017).

A GNPE é considerada doença aguda que surge entre 7 e 12 semanas após os eventos desencadeantes de infecção. Pode ocorrer a resolução espontânea rápida do quadro clínico na maioria dos pacientes, principalmente de crianças de 3 a 12 anos, ou o surgimento de quadros clínicos mais complexos com necessidade de uso de diuréticos, antibióticos, anticonvulsivantes, restrição dietética de água, sódio, potássio e proteínas e até mesmo diálise. Nestes casos de maior gravidade, principalmente em adultos, é fundamental a obtenção de diagnóstico diferencial e tratamento precoce e oportuno visando principalmente restabelecer a normalidade da taxa de filtração glomerular. Novas abordagens terapêuticas têm sido estudadas nos últimos anos, incluindo agentes específicos e com menor risco de toxicidade, incluindo os marcadores biológicos e o desenvolvimento de vacinação protetora contra padrões moleculares nefritogênicos bacteriamos e virais (COUSER WG, 2016; ROSARIO CS e JOÃO PRD, 2011).

A GNPE é atualmente considerada doença rara em países desenvolvidos e ainda representa um grande desafio devido ao risco de epidemias em países em desenvolvimento com importantes desigualdades sociais e econômicas, como o Brasil. Portanto, este trabalho de revisão bibliográfica tem como objetivo renovar e descrever os conhecimentos científicos nos últimos dez anos, com especial enfoque para os avanços na caracterização do quadro clínico, diagnóstico e tratamento da GNPE.

\section{REVISÃO BIBLIOGRÁFICA}

\section{Epidemiologia e etiologia}

Cerca de $97 \%$ dos casos de GNPE ocorrem em países em desenvolvimento devido principalmente às precárias condições higiênico-sanitárias das habitações da população de baixa renda mais sujeita as 
infecções bacterianas e outros agentes nefritogênicos com proteína $M$ na parede celular, principalmente do estreptococos beta-hemolítico do grupo A e ocasionalmente dos grupos C e G7. Nos países desenvolvidos esta doença vem reduzindo significativamente nas últimas décadas devido ao tratamento precoce das infecções e melhores condições de vida dos habitantes. Mesmo assim, mais de 470000 casos de GNPE e 5000 mortes ocorrem no mundo anualmente, com incidência de 9,5-28,5 por 100000 habitantes. Os casos podem ser esporádicos ou ocorrer em epidemia, envolvendo principalmente crianças de 5 a 12 anos com histórico de amidalite ou impetigo. O gênero masculino é mais acometido que o feminino, numa proporção de 2:1, sendo rara em crianças abaixo de 2 anos e com possibilidades de ocorrência em adultos a partir de 60 anos (BECQUET O, et al., 2009; RODRIGUEZ-ITURBE B e MUSSER JM, 2008; RAMANATHAN G, et al., 2017).

De acordo com Soares PA, et al. (2017), em condições de várias regiões brasileiras, a GNPE pode representar riscos de casos esporádicos ou até mesmo surtos epidêmicos, principalmente em áreas rurais onde se consome leite cru ou laticínios, sorvetes e outros alimentos derivados do leite sem a fiscalização das normas adequadas preconizadas pela vigilância sanitária. Esse problema epidemiológico pode se estender para amplas áreas de favelas na periferia urbana com condições consideradas péssimas de higiene, moradia e saneamento. Ao contextualizar esse cenário com os inúmeros relatos da incidência de desnutrição, anemia e parasitose intestinal em população residente no interior de vários estados brasileiros, onde são mais comuns os fatores desencadeantes de infecções de pele e GN como hábitos de consumo de alimentos in natura e não devidamente higienizados.

\section{Etiopatogenia}

Apesar dos inúmeros estudos e avanços científicos obtidos nas últimas décadas, no sentido de explicar a etiopatogenia da GNPE e de outras GN relacionadas a infecção, as teorias mais aceitas envolvem o aprisionamento de imunocomplexos circulantes (antígeno) que conduzem a formação de um complexo imunitário in situ resultante da reação de anticorpos tanto contra componentes do estreptococo como contra a estrutura do próprio glomérulo. Este complexo imunitário, passivamente aprisionado, acaba mediando o estabelecimento da lesão glomerular. Ao iniciar a formação de depósitos imunes, desencadeando uma série de alterações fisiológicas nos rins, provocam mudanças significativas da função filtrante glomerular e o acúmulo ou a liberação anormal de compostos presentes no sangue (EISON TM, et al. (2011); RAMANATHAN G, et al., 2017; YOSHIZAWA N, 2000).

De acordo com a literatura, as evidências apontam para uma doença associada a amidalite ou faringite, imunologicamente mediada, com desenvolvimento de determinadas cepas nefritogênicas, com a ação de um antígeno estreptocócico capaz de iniciar um processo imunológico que levaria à produção de anticorpos e, consequentemente, à formação de imunocomplexos nos glomérulos. Contanto, há duas vias relacionadas ao desencadeamento inflamatório de GNPE, que ainda não estão devidamente estabelecidas, uma via alternativa ou a via da lectina ligadora da manose, que atuam independente do anticorpo, o que torna a sua etiopatogenia ainda não totalmente conhecida. As deficiências tanto genéticas como adquiridas de proteínas reguladoras do complemento (como o fator $\mathrm{H}$ ) podem ter ligação com casos atípicos desta $\mathrm{GN}$ em que pacientes apresentam quadro clínico prolongado e progressivo, sem a remissão completa observada em casos pediátricos.

Existem várias teorias tentando compreender a GNPE, tendo em vista a formação de imunocomplexos in situ, para identificar o antígeno estreptocócico responsável pelo processo imunológico, em que duas são mais aceitas: teoria catiônica de Vogt (heteróloga) e teoria das neuraminidases (autóloga). Ambas tentam explicar a interação e fixação do agente infeccioso com a superfície da MBG a níveis de antígenos e proteínas e sítios específicos. Com patogenicidade determinada pela herança genética e geográfica dos pacientes, complexidade desse processo aumenta com a identificação de antígenos específicos (RODRIGUEZ-ITURBE B e MUSSER JM, 2008; YOSHIZAWA N, et al., 2004).

De acordo com Couser WG (2016) apenas a realização de mais estudos específicos poderá contribuir para definir, de forma clara, se a infecção opera apenas como fator desencadeante de um complexo de entidades sobrepostas que incluem tanto a GNPE clássica, como a atípica. Cabe elucidar ainda se o papel 
principal do mecanismo patológico seja mesmo do depósito de imunocomplexos circulantes ou de anomalia na ativação e regulação do complemento por parte do paciente em questão.

\section{Fisiopatologia}

Com a perda da integridade da MBG em função do processo inflamatório da GNPE ocorre a redução da filtração glomerular e uma diminuição da oferta de água e eletrólitos aos túbulos renais. Outra grave consequência é a passagem de elementos que normalmente não são filtrados: hemácias, leucócitos e proteínas.

Outras complicações surgem devido a menor eficiência dos capilares e do comprometimento glomerular, podendo haver a retenção de alguns compostos, como creatinina, ureia e potássio, entre outros, levando o paciente à IRA. Além disso, pode ocorrer também uma reabsorção de sódio e água, provocando a oligúria e aumento do volume extracelular circulante, resultando em edema, hipertensão arterial e congestão circulatória (BECQUET O, et al., 2009; RIBEIRO NETO JPM e PONTUAL MP, 2014; STERR AC, et al., 2007).

Embora não sejam características específicas da GNPE, os rins e seus glomérulos apresentam-se aumentados, com aspecto pálido com proliferação glomerular difusa das células mesangiais e inflamações dos espaços intersticiais em exames de microscopia ótica. Sob microscopia de imunofluorescência é possível observar depósitos irregulares com ou sem imunoglobina $G(\lg G)$ e complemento $C 3$ na MBG e no mesângio. Existe evidência de uma forma diferente de Staphylococcus associada à GNPE que induz depósitos glomerulares de imunocomplexos e seus complementos (C3), IgG, IgM e IgA. Na microscopia eletrônica podem ser verificados depósitos eletrodensos abundantes e consistentes em forma de corcova de localização tipicamente subepitelial da MBG (COUSER WG, 2016; HAHN RG e FORMAN TA, 2005; RODRIGUEZITURBE B, et al., 1998; WEHBE E, et al., 2011).

\section{Manifestações clínicas}

O quadro clínico de pacientes com GNPE se mostra bastante variável, desde assintomático até casos graves de terapia renal substitutiva. A sintomatologia renal dessa doença manifesta-se, geralmente, de 7 a 14 dias após a infecção de pele, comumente no verão (piodermite estreptocócica), ou através da via aérea superior no período de inverno (faringite estreptocócica), apresentando-se mais comumente com o início súbito de hematúria macroscópica ou microscópica, edema de membros inferiores e/ou face e hipertensão arterial. A proteinúria no início pode não ocorrer, mas geralmente apresenta-se com valores inferiores a 1g/L. Os sintomas considerados típicos incluem oligúria e acometimento moderado da função renal, podendo ocorrer, ocasionalmente, hipervolemia com edema agudo pulmonar, crise hipertensiva com encefalopatia e convulsões, e IRA, conduzindo a necessidade de diálise de urgência (HAHN RG e FORMAN TA, 2005; KASAHARA T, et al. 2001; RAMANATHAN G, et al., 2017; RIBEIRO NETO JPM e PONTUAL MP, 2014).

De acordo com Kanjanabuch T, et al. (2009), Kasahara T, et al. (2001) e Garnier A, et al. (2009) embora existam relatos de complicações renais graves como doença renal progressiva, IRA, síndrome nefrótica em aproximadamente $5 \%$ dos casos e redução leve a moderada, e de curta duração, da taxa de filtração glomerular em até $50 \%$ dos casos, associada com a GNPE, a evolução dessa doença é geralmente com quadro leve a moderado, principalmente em crianças.

A proteinúria apresenta-se com valores inferiores a $1 \mathrm{~g} / \mathrm{L}$ ou mesmo se mostrar ausente. Grande parte das alterações urinárias regridem entre 4 a 6 semanas, no entanto, em cerca de $10 \%$ dos casos a hematúria residual possa persistir por alguns meses. Nestes casos, torna-se relevante analisar a possibilidade de biópsia renal, principalmente se houver suspeita clínica de um padrão que não seja de proliferação endotelial e mesangial. Mesmo assim, a terapia renal substitutiva é considerada rara.

A ocorrência de acometimento vascular é variável, sendo que a insuficiência cardíaca congestiva pode ser observada em 15 a $50 \%$ dos casos e a hipertensão em até $90 \%$. Complicações cerebrais da hipertensão arterial, incluindo dores de cabeça, convulsões, alterações do estado mental, e alterações visuais ocorrem em 30-35\% das crianças. Porém, conforme a morbidade da doença o acompanhamento em longo prazo tornase necessário. 


\section{Diagnóstico}

Anamnese detalhada que possa detectar a ocorrência de faringite e piodermites recentes e exame físico cuidadoso podem auxiliar na identificação de sinais clínicos e manifestações extra renais. Porém, como existem vários tipos de GN, torna-se fundamental para o diagnóstico precoce e seguro da GNPE, a realização de alguns exames complementares. Destacam-se, nesse sentido, o exame do complemento sérico C3 que é fundamental para o diagnóstico diferencial dessa GN, e encontra-se diminuído em 95\% dos casos, sendo que seus níveis geralmente se normalizam em 4 a 8 semanas (RODRIGUEZ-ITURBE B e MUSSER JM, 2008).

São considerados relevantes em pacientes com suspeita de GNPE, a realização de exames de alterações urinárias, especialmente do sedimento urinário, que podem evidenciar hematúria, leucocitúria, cilindrúria. Um sangramento de origem renal pode ser indicado através de detecção de cilindros hemáticos. No início da fase aguda, os leucócitos urinários podem predominar sobre as células vermelhas do sangue. Também, os exames de bioquímica sérica podem mostrar se os níveis de ureia e creatinina tiveram elevação significativa, indicando que o paciente sofre de doença renal crônica em evolução para IRA. A hematologia pode evidenciar anemia discreta dilucional advinda da hipervolemia. Além disso, de acordo com a evolução da doença, do sorotipo infectante e o local de infecção, o título de antiestreptolisina pode se mostrar elevado (RIBEIRO NETO JPM e PONTUAL MP, 2014; STERR AC, et al., 2007).

\section{Prevenção}

Os cuidados básicos de higiene pessoal, principalmente banho e lavagem frequentes das mãos, e atenção para possíveis sinais de piodermites ou mesmo manifestações leves de faringites podem auxiliar na prevenção de GNPE. As manifestações de infecção de pele, especialmente em crianças, devem merecer atenção especial dos pais e busca por atendimento médico. Situações em que as condições de moradia são muito precárias, em que se soma falta de saneamento básico e higiene, principalmente de grande parte da população de baixa renda, cenário comum de países em desenvolvimento, como o Brasil, o ideal seria o desenvolvimento de políticas públicas de orientação para os riscos de ocorrências pontuais ou mesmo de epidemias de GN. Campanhas educativas sobre higienização e os riscos de consumo de alimentos crus são peças fundamentais em programas de prevenção. Pesquisas de vacinas têm sido propostas e conduzidas, porém ainda não estão disponibilizadas para atender especialmente a indivíduos que vivem em áreas endêmicas (BATZLOFF M, et al., 2004; KOTLOFF KL e DALE JB, 2004).

\section{Tratamento}

Cada caso de GNPE precisa ser analisado de forma específica em termos de tratamento, considerando vários fatores: quadro clínico apresentado, idade, presença ou não de complicações e até mesmo as condições socioculturais e econômicas do paciente. Essa personalização no atendimento e terapêutica relaciona-se diretamente às possibilidades atualmente disponíveis de apresentar várias formas de tratamentos individualizados. De modo geral, a orientação adotada é sempre que possível preferir o tratamento em domicílio, principalmente quando o paciente apresenta quadro clínico leve a moderado, restringindo a internação hospitalar para situações de gravidade como oligúria intensa, insuficiência cardíaca ou encefalopatia hipertensiva (RIBEIRO NETO JPM e PONTUAL MP, 2014; STERR AC, et al., 2007).

De acordo com Rodriguez-Iturbe B e Musser JM (2008) e Srivastava RN (1999), alguns cuidados gerais podem auxiliar no tratamento de GNPE, destacando-se: o repouso, principalmente em casos com algum tipo de complicação, passando por atividades físicas moderadas à medida que o paciente apresente melhora do quadro clínico; adoção de dieta restritiva principalmente com redução na ingestão de líquidos e sódio (20 $\mathrm{mL} / \mathrm{kg} / \mathrm{dia}$ e menos de $2 \mathrm{~g}$ de $\mathrm{NaCl} / \mathrm{m}^{2} / \mathrm{dia}$, respectivamente), principalmente se for observado edema, hipertensão e oligúria. É recomendável também limitar a ingesta de proteínas por um período, até que os níveis de ureia e creatinina no sangue voltem aos níveis considerados normais.

O tratamento medicamentoso pode envolver o uso de antibióticos, especialmente penicilina, visando erradicar o estreptococo ou outras cepas nefritogênicas. Tratando-se de sobrecarga hídrica e hipertensão arterial, o uso de diuréticos deve ser feito, sendo relevante também em casos com gravidade quando se 
observa congestão cardiocirculatória, oligoanúria e a hipertensão arterial não cede, mesmo com a restrição dietética.

A prescrição de hipotensores como hidralazina, nifedipina e anlodipina se faz necessária quando ocorre a persistência da hipertensão, mesmo com o desparecimento do edema e oligúria. A introdução dos inibidores da enzima conversora da angiotensina (IECA) já foi relatado com sucesso no controle da pressão arterial nesses casos.

Existe, no entanto, uma ressalva importante quando há lesão renal aguda já instituída de forma mais intensa, podendo aumentar o risco de hipercalemia e queda acentuada da taxa de filtração glomerular. Anticonvulsivantes como diazepan e outros podem ser prescritos em casos de convulsões associadas a encefalopatia hipertensiva (MARQUES VP, et al., 2010; PONTUAL MP e MACIEL MSV, 2004; RODRIGUEZITURBE B, et al., 1998).

De acordo com Vinen CS e Oliveira DB (2003), há situações em que o paciente com GNPE crescente necessita de diálise, especialmente quando se observa: hipervolemia grave (anasarca, edema agudo de pulmão), distúrbios hidroeletrolíticos graves, acidose metabólica ( $\mathrm{pH}$ menor que 7,0), intoxicações específicas, uréia acima de $200 \mathrm{mg} / \mathrm{dL}$ e sintomas neurológicos. O diagnóstico precoce desta GN pode auxiliar no sentido de se evitar esse quadro clínico mais grave ou tornar menor o período de diálise do paciente, reduzindo os riscos de complicações e a fragilização das condições de saúde.

De acordo com Couser WG (2016), em conjunto ao desenvolvimento de vacinas contra padrões moleculares de agentes infectantes, mostra-se de grande potencial as pesquisas que visam a obtenção de biomarcadores para a identificação de pacientes com GNPE atípica. Isto poderá viabilizar a adoção de tratamento precoce de tais indivíduos com vários produtos biológicos, especialmente de inibidores do complemento sérico $\mathrm{C} 3$, visando a interrupção da evolução da inflamação e de suas consequências a longo prazo na recuperação espontânea completa da doença.

\section{Evolução e prognóstico}

De acordo com Kasahara T, et al. (2001) e Steer AC (2007), na primeira ou segunda semana após o estabelecimento da GNPE, na maioria dos casos, já é possível observar a diminuição do edema e da hipertensão, bem como o desaparecimento da hematúria macroscópica, apesar da microscópica poder persistir por até 18 meses.

Apesar da existência de relatos de casos com complicações renais graves (doença renal progressiva e IRA), geralmente o prognóstico deste tipo de GN é considerado benigno em crianças e adolescentes, sendo raro os casos de óbitos e apenas $5 \%$ tornam-se crônicos.

Mesmo assim é fundamental a atenção da vigilância epidemiológica no sentido de melhor conhecer as variações cíclicas de GNPE dentro de uma mesma população, bem como o histórico de ocorrências de epidemias, a fim de se evitar subnotificações e melhorar a confiabilidade dos dados de sua incidência e da gravidade dos casos registrados.

\section{CONSIDERAÇÕES FINAIS}

Considerando os vários tipos de glomerulopatias, a GNPE merece destaque especial em se tratando das condições socioeconômicas brasileiras, devido ao seu potencial de ocorrência de forma esporádica ou em surtos epidêmicos na população de baixa renda. Embora a maioria dos casos envolva crianças e adolescentes e geralmente apresentam prognósticos favoráveis, mais atenção precisa ser dada para a ocorrência em adultos e idosos, no sentido de evitar que se tornem doença crônica e endêmica. Os avanços obtidos nos últimos anos em termos de diagnóstico diferencial e tratamento da GNPE podem auxiliar os profissionais de saúde e as autoridades a vencer este desafio e na adoção de políticas públicas que atuem mais na prevenção das doenças precursoras, tais como faringite e infecções de pele. 


\section{REFERÊNCIAS}

1. BATZLOFF M, et al. Preclinical evaluation of a vaccine based on conserved region of $M$ protein that prevents group A streptococcal infection. Indian Journal Medicine Research. 2004;119(suppl):104-107.

2. BECQUET O, et al. Acute poststreptococcal lomerulonephritis in children of French Polynesia: a 3-year retrospective study. Pediatric Nephrology. 2009;25:275-280.

3. COUSER WG. Patogênese e tratamento da glomerulonefrite - uma atualização. Jornal Brasileiro Nefrologia. 2016;38(1):107-122.

4. EISON TM, et al. Post-streptococcal acute glomerulonephritis in children: clinical features and pathogenesis. Pediatric Nephrology. 2011;26:165-180.

5. GARNIER A, et al. Glomérulonéphrite aiguë postinfectieuse. Postinfectious acute glomerunephritis. Néphrologie \& Thérapeutique. 2009;5:97-100.

6. HAHN RG, FORMAN TA. Evaluation of Poststreptococcal Illness. American Family Physician. 2005;71(10): 19491954.

7. KANJANABUCH T, et al. An update on acute postinfectious glomerulonephritis worldwide. National Review Nephrology. 2009;5:259-269.

8. KASAHARA T, et al. Prognosis of acute poststreptococcal glomerulonephritis (APSGN) is excellent in children, when adequately diagnosed. Pediatrics International. 2001;43:364-367.

9. KOTLOFF KL, DALE JB. Progress in group A streptococcal vaccine development. Pediatric Infection Disease Journal. 2004;23:765-766.

10. MARQUES VP, et al. Glomerulonefrite aguda após infecção de vias áereas superiores ou pele: análise descritiva de 82 pacientes entre 14 e 64 anos de idade. Jornal Brasileiro Nefrologia. 2010;32(3):237-241.

11. PONTUAL MP; MACIEL MSV. Glomerulonefrite difusa aguda pós-estreptocócica. In: ALVES BGJ, FERREIRA SO, MAGGI SR (eds.). Fernando Figueira pediatria (Imip). 3.ed. Rio de Janeiro: Medsi; 2004. p.797-801.

12. RAMANATHAN G, et al. Analysis of clinical presentation, pathological spectra, treatment and outcomes of biopsyproven acute postinfectious glomerulonephritis in adult indigenous people of the Northern Territory of Australia. Nephrology. 2017; 22(5):403-411.

13. RIBEIRO NETO JPM, PONTUAL MP. Glomerulonefrite difusa aguda pós-estreptocócica. In: CAMPOS JUNIOR D, BURNS DAR (org.). Tratado de pediatria. 3.ed. Barueri: Manole; 2014. 1713-1718p.

14. RODRIGUEZ-ITURBE $B$, et al. Antibody to streptococcal zymogen in the sérum of patients with acute glomerulonephritis: a multicentric study. Kidney International. 1998;54:509-517.

15. RODRIGUEZ-ITURBE B, MUSSER JM. The current state of poststreptococcal glomerulonephritis. Journal American Society Nephrology. 2008;19:1855-1864.

16. ROSÁRIO CS, JOÃO PRD. Glomerulonefrite aguda pós-infecciosa: relato de caso. Revista Médico Residente. 2011; 13(3):206-210.

17. SOARES PA, et al. Epidemia de nefrite por Streptococcus equi subsp. zooepidemicus: estudo de caso-controle no município de Monte Santo de Minas, Minas Gerais, 2013. Epidemiologia Serviço Saúde. 2017;26(2):405-412.

18. SRIVASTAVA RN. Acute Glomerulonephritis. Indian Journal Pediatric. 1999;66:199-205.

19. STEER AC, et al. Group A streptococcal infections in children. Journal Paediatric Children Health. 2007;43: 203-213.

20. VINEN CS, OLIVEIRA DB. Acute glomerulonephritis. Postgraduate Medicine Journal. 2003;79: 206-213.

21. WEHBE E, et al. IgA-dominat Staphylococcus infection-associated glomerulonephritis: case reports and review of the literature. Nephrology Dialysis Transplantation. 2011;4: 181-185.

22. YOSHIZAWA N. Acute Glomerulonephritis - Review Article. Internal medicine. 2000;39(9):687-689.

23. YOSHIZAWA N, et al. Nephritis associated plasm in receptor and acute post streptococcal glomerulonephritis: characterization of the antigen and associated immune response. Journal American Society Nephrology. 2004; 15:1785-1793. 